\title{
A fast method to prepare mechanically strong and
} water resistant lignocellulosic nanopapers

$$
\text { Jatin Sethi }{ }^{1} \text {, Miikka Visanko }{ }^{1}, \text { Monika Österberg }{ }^{2} \text {, and Juho Antti Sirviö }{ }^{1^{*}}
$$

1. Fibre and Particle Engineering Research Unit, University of Oulu, Oulu, Finland.

2. Department of Bioproducts and Biosystems, Aalto University, Helsinki, Finland.

\section{Abstract}

This study covers a green method to prepare hybrid lignocellulosic nanopapers by combining wood nanofibres (WNFs) and cellulose nanofibres (CNFs). The WNFs and CNFs behave synergistically to compensate for the drawbacks of each other resulting in enhanced hybrid nanopapers. The draining time of hybrid nanopapers was improved by up to $75 \%$ over CNF nanopaper, and the mechanical properties (modulus, strength and elongation) were respectively improved up to $35 \%$, $90 \%$ and $180 \%$ over WNF nanopaper. Additionally, the water resistance of hybrid nanopapers was considerably improved with a water contact angle of $95^{\circ}$; the neat CNF nanopaper had a contact angle of $52^{\circ}$. The morphology of nanopapers, studied by electron microscopy, indicated that lignin acts as a matrix, which binds the nanofibres together and makes them impervious to external environmental factors, such as high humidity. The reported hybrid nanopapers are $100 \%$ biobased, prepared by a simple and environmentally friendly processing route. Reported hybrid nanopapers can be used in novel applications such as gas barrier membranes and printable electronics.

\section{Keywords}

Hybrid nanopapers; Wood nanofibres; Cellulose nanofibres; Dewatering; Water resistance;

Thermal properties. 

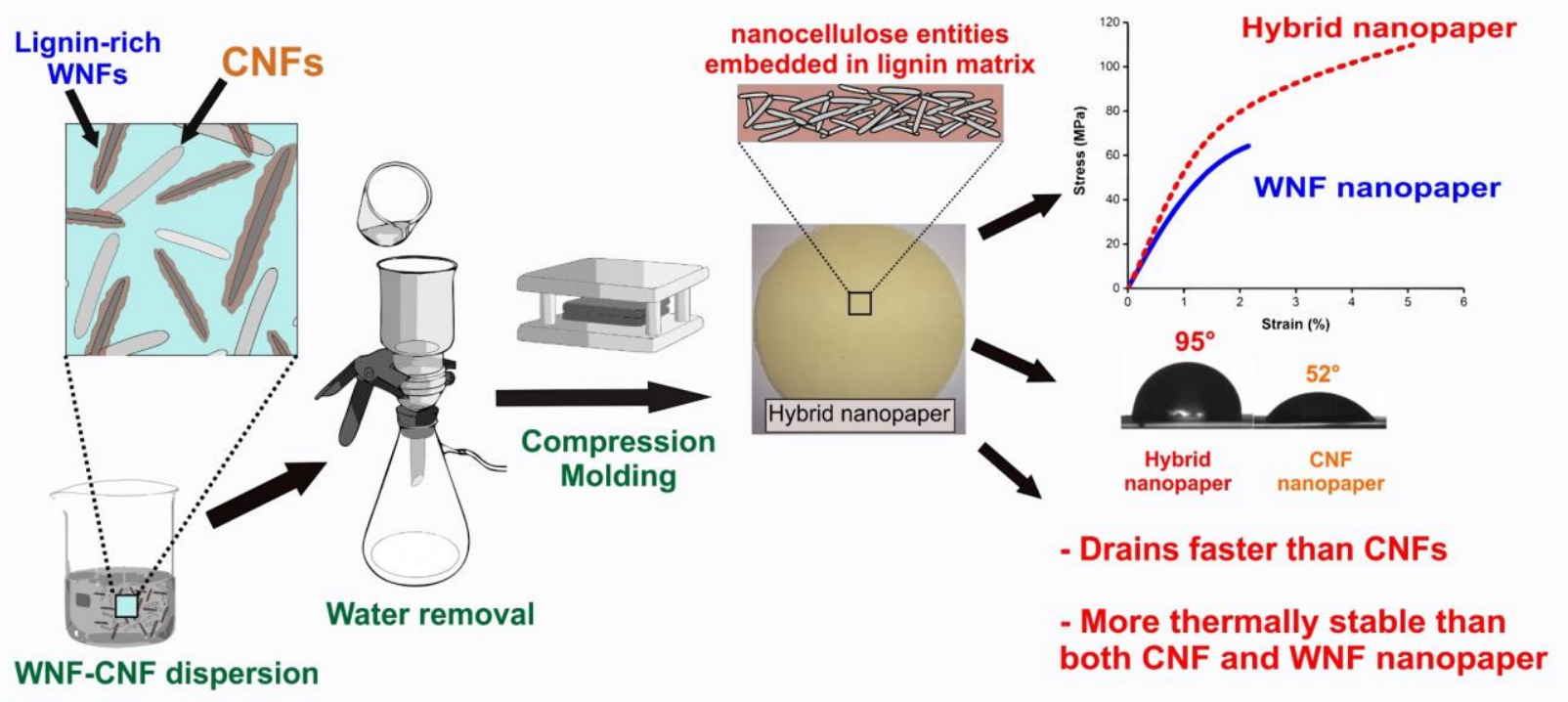

\section{Introduction}

28 With the advent of nanotechnology, novel and intriguing functional materials have been coming

29 into the spotlight. A thin, freestanding film called as nanopaper is one such material. Nanopapers

30 are formed from highly concentrated, tightly packed nanoparticles, such as carbon nanotubes

31 (CNTs), carbon nanofibres, graphene, clay nanoplatelets or cellulose nanofibres (CNFs) (Huang,

2017). Their unique and distinctive properties make them stand out from other conventional

material. Nanopapers prepared from carbon-based nanomaterials have excellent electrical

34

conductivity (Salajkova, Valentini, Zhou, \& Berglund, 2013). Nanopapers based on clay

nanoplatelets (CNPs) result in excellent barrier properties and thermal stability (Sehaqui, Kochumalayil, Liu, Zimmermann, \& Berglund, 2013). CNF nanopapers, on the other hand, are known for their extreme toughness (M. Henriksson, Berglund, Isaksson, Lindström, \& Nishino, 2008)(Deng, Huang, Zhou, Chen, \& Fu, 2016). Additionally, CNF nanopapers have a low thermal expansion coefficient (similar to glass) and a higher dielectric constant and thermal durability than most plastics; yet, it is foldable like paper (Yagyu, Ifuku, \& Nogi, 2017). Such a variety of functional properties make them suitable for multifunctional and high-end applications such as electronic 
displays (Sehaqui, Zimmermann, \& Tingaut, 2014), flexible electronics (Koga et al., 2014), organic solar cells (Yagyu et al., 2017) and lithium ion batteries (Chun, Lee, Doh, Lee, \& Kim, 2011).

CNF nanopaper has been a centre of attention for its simple preparation and lightweight structure and due to the renewable nature of the raw material. CNFs are usually prepared by disintegrating bleached pulp in stone grinders or microfluidisers. They usually have a thickness of a few nanometers and a length of a few micrometres (Kargarzadeh, Ahmad, Thomas, \& Dufresne, 2017). CNF nanopapers are prepared from CNF suspensions by using vacuum filtration for water removal through vacuum filtration, followed by drying the wet CNF mat. They are transparent (partially or fully) with ultrastrong mechanical characteristics. They can have an elastic modulus of tens of GPa and tensile strength of few hundred MPa (M. Henriksson et al., 2008; Sehaqui et al., 2012). Such a high degree of mechanical stiffness combined with the ease of preparation is unheard of in the case of an artificial polymeric material, and a green renewable material providing such properties is an unprecedented advantage. However, despite their numerous advantages, CNF nanopapers are not problem-free. The primary challenge is their affinity for water, which affects both the preparation and long-term use of the nanopapers. Usually, cellulose nanofibres have the allomorph of cellulose which mostly contains (110) surfaces that are highly decorated with protruding hydroxyl groups (Mazeau \& Rivet, 2008). This abundance of hydroxyl groups makes them hydrophilic and, as a result, CNFs retain a lot of water, which is hard to remove during filtration. The dewatering time usually lasts up to a few hours (Iwamoto, Nakagaito, Yano, \& Nogi, 2005), which makes them difficult to commercialise. Advanced methods that drain water in $0.5-1$ hours have been reported but they are few in number (Österberg et al., 2013; Sehaqui, Liu, Zhou, \& Berglund, 2010). In addition, water causes problems with the functionality of CNF nanopapers as it weakens the finished product. A loss of $95 \%$ in the modulus was reported when the nanopaper was in contact with water (Sehaqui et al., 2014). Lucenius et al. also reported a loss of $80-90 \%$ in the mechanical properties of wet CNF nanopapers (Lucenius, Parikka, \& Österberg, 2014). Even in the presence of high relative humidity (or $\mathrm{RH}$; specifically, above $70 \% \mathrm{RH}$ ), CNF nanopapers' properties deteriorate rapidly (Benítez, Torres-Rendon, Poutanen, \& Walther, 2013; Sethi et al., 2018). 
Although some post-chemical modification has been proposed to improve the tolerance of nanopapers towards the water and humidity (Lucenius et al., 2014; Sehaqui et al., 2014), simplified and eco-friendly methods are desired to deal with the dewatering time and water and humidity tolerance of CNF nanopapers before they can be mass-produced.

CNFs are extracted from lignocellulosic biomass in two principal steps. Firstly, a harsh chemical pre-treatment is done to remove the lignin and hemicellulose. The origin of this process comes from the paper industry, which requires bleaching (cleaning) of the pulp before papermaking. Secondly, the treated (bleached) pulp is then severely ground to separate individual CNFs from the after cellulose, and comprises up to $40 \%$ of plant biomass (G. Henriksson, 2017). It is necessary for proper load distribution and for maintaining the integrity of the wood. Additionally, lignin provides water resistance to the wood. Finally, the removal of lignin is an expensive process as it is usually done by kraft pulping, which utilises expensive and hazardous chemicals. Therefore, it is of interest to research lignin-rich wood nanofibres (WNFs) as a potential raw material for making studies have been reported on the topic (Herrera et al., 2018; Nair \& Yan, 2015; Rojo et al., 2015; Visanko et al., 2017). Nanopapers made from WNFs offer certain advantages such as quicker preparation time and improved water resistance. However, WNF nanopapers have lower mechanical properties ( $25 \%$ less modulus, $45 \%$ less yield strength) and are brittle (20\% less elongation) when compared to CNF nanopaper (Visanko et al., 2017).

Nanopapers prepared from either WNF or CNF have distinct advantages, yet they suffer from inherent drawbacks, which need to be addressed. Interestingly, the advantages and drawbacks seem to be mutually exclusive. CNF nanopapers are tough but it takes hours to prepare them and they are extremely weak in the presence of moisture. WNF nanopapers are prepared quickly and they have high water resistance, but they are brittle. Therefore, it makes sense to prepare a hybrid 
different kinds of nanostructured particles to make a hybrid multiphase material is a well-known, fruitful way of improving the properties of nanopapers, where each ingredient complements the other. In CNT-CNF hybrid nanopaper, CNTs imparted the electrical conductivity, and CNFs provided a water-based processing route, along with a cheap, high strength matrix (Salajkova et al., 2013). Similar synergistic behaviour was observed between CNP-CNF nanopapers. CNPs imparted fire retardancy and barrier properties, and CNFs contributed to high elongation, which was missing in nanopaper made exclusively from CNPs (Liu, Walther, Ikkala, Belova, \& Berglund, 2011). The current work is inspired by a similar principle. We hypothesised that an intricate WNFCNF interpenetrating network in a nanopaper would allow each material to make up for other's deficiencies. WNFs would benefit from the integral CNF network, which has extraordinary dry mechanical properties such as high modulus and elongation. In return, the CNF network would benefit from the binding properties of the WNF network, water resistance, and quicker draining time. We report a novel method to prepare lignin-rich hybrid nanopaper by using a mixture of CNF and lignin WNF suspensions in water.

This study provides a simplistic and industrially adaptable method of preparing lignin rich hybrid nanopaper and discusses the advantages of lignin-rich hybrid nanopapers. The primary aim of this study was to create and study a hybrid nanopaper prepared from WNFs and CNFs. WNFs with $27 \%$ lignin content were prepared through high temperature grinding and combined with CNFs in ratios of 9:1, 3:7, 5:5 and 7:3. The neat WNF and neat CNF films were also prepared as references. The morphology was studied with the help of scanning electron microscopy. Mechanical properties were characterised by the tensile testing (dry and wet). The effect of humidity on mechanical properties was studied by dynamic mechanical analysis. Thermal gravimetric analysis was used to study thermal properties, and contact angle measurements were used for evaluating the water repellency of the nanopapers. This research, for the first time, reports a hybrid nanopaper prepared from WNFs and CNFs, which is $100 \%$ bio based. Additionally, this is a first attempt that mentions the drawbacks of WNF nanopaper and improves it with an eco-friendly method. 


\section{Materials and methods}

\subsection{Preparation of nanofibres}

WNFs were prepared from unbleached spruce groundwood pulp (never dried), which was provided by Stora Enso (Veitsiluoto, Finland). To prepare WNFs, the high temperature thermomechanical grinding of wood pulp was done in Masuko super mass collider (MKCA6-2 J CE; Masuko Sangyo, Japan) according to the method reported by (Visanko et al., 2017). Briefly, 1.5 wt.-\% of wood pulp (4kg total weight) was heated until the temperature reached $95^{\circ} \mathrm{C}$. The heated pulp was repeatedly fed in grinding disks (10-inch diameter). The outcome from the grinder was collected, heated back to $95^{\circ} \mathrm{C}$ and fed back into the grinder. The distance between the grinding disks was gradually decreased. The pulp was ground once through $0 \mu \mathrm{m}$ and once through $-50 \mu \mathrm{m}$, and fifteen times through $-100 \mu \mathrm{m}$ disk clearance. On the completion of grinding, the WNF suspension was collected and stored at a temperature of $4^{\circ} \mathrm{C}$. For more details about WNFs and its characteristics, the readers are advised to follow the earlier publication (Visanko et al., 2017).

Cellulose nanofibres were prepared from softwood sulphite pulp, also provided by Stora Enso (Veitsiluoto, Finland). For grinding, they were also repeatedly fed in the Masuko super mass Collider (MKCA6-2 J CE; Masuko Sangyo, Japan). The pulp was passed three times from -20 $\mu$ m, four times from $-40 \mu \mathrm{m}$, five times from $-60 \mu \mathrm{m}$, and seven times from $-90 \mu \mathrm{m}$ clearances. On the completion of grinding, the WNF suspension was collected and stored at a temperature of $4^{\circ} \mathrm{C}$.

\subsection{Preparation of nanopapers}

To prepare the hybrid nanopapers, the WNF and CNF suspensions were added to a glass beaker and diluted to a concentration of 0.2 wt.- $\%$, according to the formulations presented in Table 1 . The WNF-CNF suspension was mixed using an Ultra-Turrax homogeniser, at $10000 \mathrm{rpm}$ for $5 \mathrm{~min}$, and degassed under a vacuum of $-70 \mathrm{kPa}$ for $15 \mathrm{~min}$ to remove air bubbles. Finally, the water was drained by vacuum filtering the WNF-CNF suspension through a Durapore $0.65 \mu$ m polyvinylidene fluoride membrane (M. Henriksson et al., 2008; Sethi et al., 2018). The vacuum applied was $-70 \pm$ 
$5 \mathrm{kPa}$. The draining was assumed complete when the time difference between the fall of two consecutive water drops from the funnel was 30 seconds. After the draining was complete, the WNF-CNF wet mat was carefully peeled from the membrane and stacked between two steel meshes (70 $\mu \mathrm{m}$ pore size) and blotting paperboards. The whole assembly was compression moulded between hotplates at $150^{\circ} \mathrm{C}$ for 3 min at $2 \mathrm{MPa}$ and $15 \mathrm{~min}$ at $50 \mathrm{MPa}$. The samples are coded according to a ratio of WNF to CNF. For example, 70WNF30CNF represents the sample that has 70 wt.- $\%$ WNF and 30 wt.- $\%$ CNF. 100WNF and 100CNF represents the neat WNF and CNF nanopapers respectively. The grammage of nanopapers was 100-120 grams per square meter.

The cross-section and surface morphology of reference and hybrid nanopapers was studied by field emission scanning electron microscopy (FE-SEM) using a Zeiss Ultra Plus electron microscope (Oberkochen, Germany). Before imaging, the samples were coated with a thin layer of platinum. The samples were scanned with an electron beam accelerated at a voltage of $5 \mathrm{kV}$, and an in-lens detector was used to collect the signals.

Table 1. Formulation of WNFs and CNFs for the preparation of hybrid nanopaper

\begin{tabular}{cccccccc}
\hline & \multicolumn{7}{c}{ Samples } \\
\cline { 3 - 8 } \multicolumn{1}{c}{ Materials } & & 100WNF & 10CNF & 30CNF & 50CNF & 70CNF & 100CNF \\
\hline \multirow{2}{*}{ WNF suspensions } & WNF & 0.20 & 0.18 & 0.14 & 0.10 & 0.06 & - \\
& Water* & 7.8 & 7.0 & 5.4 & 3.9 & 2.3 & - \\
\hline \multirow{2}{*}{ CNF suspension } & CNF & - & 0.02 & 0.06 & 0.1 & 0.14 & 0.2 \\
& Water* & - & 1.0 & 3.1 & 5.2 & 7.2 & 10.4 \\
\hline Deionized water** & & 92.0 & 91.8 & 91.2 & 90.7 & 90.2 & 89.4 \\
\hline & Total & 100 & 100 & 100 & 100 & 100 & 100 \\
\hline
\end{tabular}

$16{ }^{* *}$ Deionised water added to dilute the CNF suspension

\subsection{Characterisation of nanopapers}

168 Water contact angles were determined using the sessile drop method. A 6.5 $\mu \mathrm{L}$ Milli-Q water droplet was placed on the surface of the samples, and 60 images of the droplet at the rate of 1 image per second were collected using a CAM 200 contact angle meter (KSC instruments, 
171 Finland). The static contact angle of each image was determined by a Young-Laplace equation estimation of the drop shape using the software provided by the manufacturer. An average of all 60 values was calculated and assumed as the contact angle of the surface. Three measurements at different positions on each sample were conducted, and the average value along with the standard deviation is reported.

To measure water absorption, the reference and hybrid nanopapers were dipped in distilled water for a prolonged period. The wet sample was periodically removed from the water after $10 \mathrm{~min}, 1$ hour, and 24 hours and then weighed on an analytical balance after blotting the excess water with a dry tissue paper. The results are expressed by an increase in weight as a percentage gain.

The wet and dry mechanical properties were evaluated with tensile testing using Zwick Roell universal test machine (Ulm, Germany). The samples were cut with scissors into50 mm x $5 \mathrm{~mm}$ rectangles, and stored in ambient conditions $\left(23^{\circ} \mathrm{C}\right.$ and $\left.55 \% \mathrm{RH}\right)$ for at least 48 hours before testing. Stress-strain curves were recorded using a load cell $1 \mathrm{kN}$ at the crosshead speed of 5 $\mathrm{mm} / \mathrm{min}$. The initial separation between the grips was $20 \mathrm{~mm}$. The elastic modulus was determined by calculating the slope of the stress-strain curve in the linear region and the yield strength was determined as the point of intersection of the stress-strain curve and offset line originating from a $0.2 \%$ co-ordinate. The results are reported as an average of at least 5 strips per sample. For wet tensile tests, the method reported by Sehaqui et al was used (Sehaqui et al., 2014). In short, a 50 $\mu$ drop was gently placed in the middle of strips. The drop was gently wiped after 60 seconds and the stress strain curves were recorded. The results are reported for an average of at least 3 samples.

The effect of humidity on the nanopapers' mechanical properties was determined using a dynamic mechanical analysis (DMA) Q800 equipped with a RH accessory (TA Instruments, New Castle, USA) in strain mode (amplitude $10 \mu \mathrm{m}$ and frequency $1 \mathrm{~Hz}$ ). The samples were first equilibrated at $95 \%$ at a rate of $1 \%$ /minute. Finally, the $\mathrm{RH}$ was maintained at $95 \%$ for 1 hour to determine the 
equilibrium value of the storage modulus. The temperature was maintained at $30^{\circ} \mathrm{C}$ for the entire duration of the test.

The thermal stability of the nanopapers was determined by a Netsch STA 409 PC (Germany). The samples were heated from room temperature to $800^{\circ} \mathrm{C}$ (heating rate $10^{\circ} \mathrm{C} / \mathrm{min}$ ) under a nitrogen atmosphere (flow rate: $60 \mathrm{ml} / \mathrm{min}$ ), and the weight loss was recorded.

The crystalline structure of the sample was determined by Wide-angle X-ray diffraction (XRD). The measurements were recorded using a Rigaku SmartLab $9 \mathrm{~kW}$ rotating anode diffractometer (Japan) using a Co Ka radiation (40 kV, 135 mA; k = $1.79030 \AA ̊$. Bragg's angle $(2 \theta)$ was varied from $10^{\circ}$ to $50^{\circ}$, with a step width of $0.02^{\circ}$ (scanning speed $=2^{\circ} \mathrm{min}^{-1}$ ). The degree of crystallinity (Crl) was calculated from the peak intensity of the main crystalline plane (200) diffraction $\left(I_{200}\right)$ which was at $26.2^{\circ}$ and from peak intensity at $22^{\circ} \mathrm{C}$, which is associated with amorphous fraction of cellulose $\left(\mathrm{I}_{\mathrm{am}}\right)$ (French, 2014), according to Equation 1:

$$
\operatorname{CrI}=\left(\frac{\mathrm{I}_{200}-\mathrm{I}_{\mathrm{am}}}{\mathrm{I}_{200}}\right)
$$

Equation 1

\section{Results and discussions}

\subsection{Draining time and ease of nanopaper preparation}

The 100WNF nanopaper was the fastest to drain (in $15 \mathrm{~min}$ ). On the other hand, 100CNF took almost 120 min to drain. The draining time is one of the most significant challenges hindering the use of CNFs in commercial applications. Clearly, if an intermediate process takes 120 min, nanopapers are unlikely to be produced on an industrial scale. It was found that by replacing a part of the hydrophilic CNFs with hydrophobic WNFs, the draining time can be considerably decreased. The hybrid nanopapers drained in a time that was in-between the draining time of 100WNF and $100 \mathrm{CNF}$, closer to $100 \mathrm{WNF}$. The draining times for nanopapers are presented in Figure 1. To summarise, $70 \mathrm{WNF} 30 \mathrm{CNF}$ took $35 \mathrm{~min}, 50 \mathrm{WNF} 50 \mathrm{CNF}$ took $55 \mathrm{~min}$, and 30WNF70CNF took 65 min. Interestingly, the partial replacement of CNF with WNF improved the draining time in contrast to the neat CNF nanopaper. For example, 30WNF70CNF should have theoretically drained in 90 
min (determined by rule of mixtures). Experimentally, it drained in $65 \mathrm{~min}$, which is an improvement of $28 \%$. In the case of others (70WNF30CNF and 50WNF50CNF), the improvement was around $20-25 \%$. The reason behind this needs to be studied further.

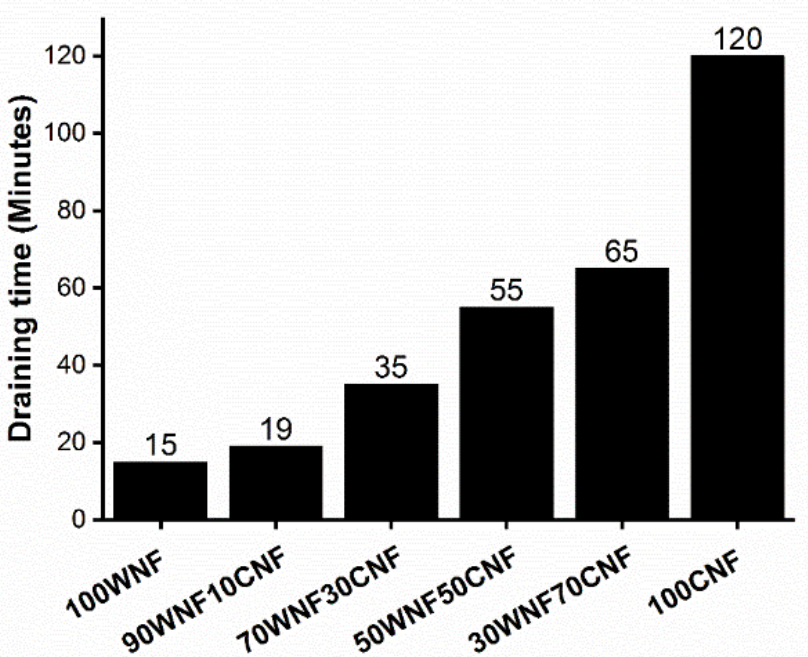

Figure 1. Draining time of references and hybrid nanopapers; $100 \mathrm{WNF}$ drained in $12 \%$ of time taken by $100 \mathrm{CNF}$

However, 100WNF, after draining swiftly, performed poorly in preparation ease. Wet 100WNF film disintegrated easily while peeling from the polyvinylidene fluoride membrane after draining, making the job tedious. The photographic image of 100WNF is shown in Figure S1 Supplementary file, which have cracks on the edges due to disintegration while peeling. The reason behind this is the lack of cohesion due to the deterioration of hydrogen bonding among the wet WNFs. It has been reported that, when wet, hydrogen bonding plays a role in strength (Tenhunen et al., 2016). WNFs are covered by lignin, which can form hydrogen bonds (Kubo \& Kadla, 2005), but not as extensively as CNFs which have a large number of hydroxyl groups. The addition of CNFs to the hybrid nanopapers strengthened the wet film, and it maintained its structure while peeling. The photographic image of a hybrid nanopaper (50WNF50CNF) is provided in Figure S1 of the supplementary file, which does not show any cracking. This difference is an added advantage of hybridization. 


\subsection{Morphology of nanopapers}

240 When observed under an electron microscope, 100WNF has a levelled surface (Figure 2a). No 241 fibrillar entities are visible showing that lignin has melted and fused to form a uniform film under the 242 influence of high pressure and temperature (Wang, Hu, \& Zeng, 2012). On the other hand, the 243 surface of $100 \mathrm{CNF}$ has an intertangled web like structure, where fibres are visible as individual 244 entities (Figure 2c), which is common for nanopaper surfaces. Finally, 50WNF50CNF has 245 characteristics of both; nanofibres are visible yet embedded in the lignin matrix (Figure 2b). The 246 structure can be assumed to be an anisotropic composite where CNFs are randomly distributed 247 within the lignin matrix. Additionally, it is apparent that the dispersion of CNFs within WNFs is 248 uniform, validating the preparation method. The presence of lignin in hybrid nanopapers are is 249 confirmed by FTIR, which are presented in Figure S2. A peak at $1510 \mathrm{~cm}^{-1}$, which represents the 250 aromatic rings of lignin, can be clearly observed in 100WNF and 50WNF50CNF. On the other 251 hand, no such peak is present in 100CNF nanopaper.
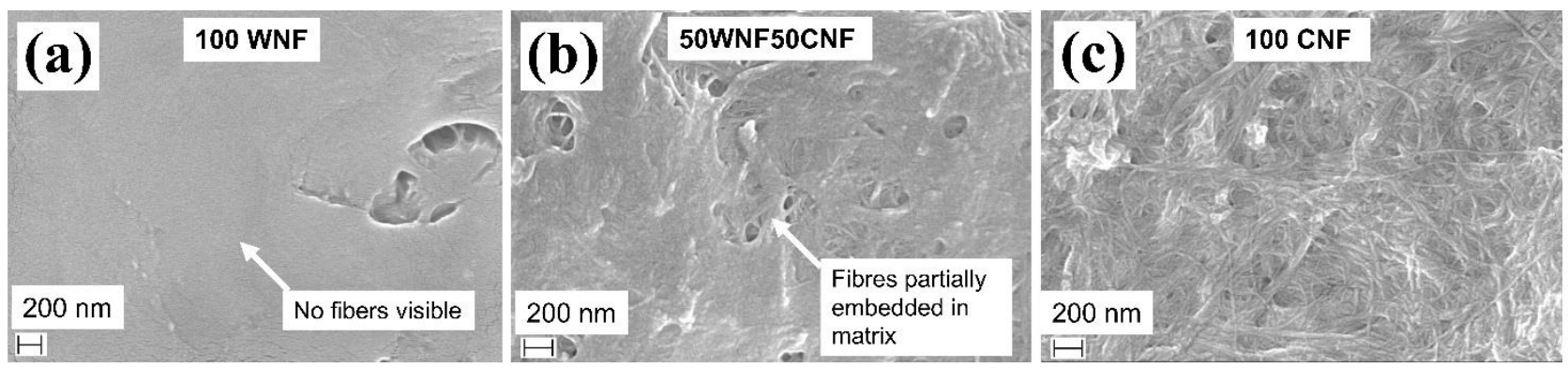

Figure 2. Surface micrographs from FESEM imaging of surface of $100 \mathrm{WNF}, 50 \mathrm{WNF} 50 \mathrm{CNF}$, and $100 \mathrm{CNF}$. No fibres are visible in 100WNF indicating the fusion of lignin. 50WNF50CNF have fibres partially embedded in lignin, and 100CNF fibres are clearly visible on the surface.

The effect of lignin was more pronounced in the images of fractured cross-sections (Figure 3). As shown in Figure $3 \mathrm{~b}$ and 3c,100CNF and 50WNF50CNF have a layered structure, which is a known characteristic of cellulosic nanopaper (M. Henriksson et al., 2008). On the other hand, in 100WNF,

259 the layers are not plainly noticeable, and the entire structure appears to be tightly glued (Figure $2603 a$ ), resulting in the brittle nature of 100WNF (Figure 4). Additionally, no visible nanofibres that 261 would be protruding from the fractured surface were seen in 100WNF (Figure 3a magnified). 
263 the layer during the fracture (Figure 3c magnified). This sliding is responsible for the ductile 264 fracture and toughness (M. Henriksson et al., 2008). The slippage of individual fibres can also be 265 observed in 50WNF50CNF (Figure 3b), which contributed to its ductility (Figure 4). Additionally, in $26650 W N F 50 C N F$, the CNFs seem to be embedded well in the lignin matrix and appear to be 267 uniformly distributed. The entire composite-like morphology of 50WNF50CNF can be imagined as 268 an interpenetrating network of CNFs and nanocellulosic entities from WNFs, with lignin acting as a 269 matrix. The benefits of this morphology are discussed in more detail in the mechanical properties 270 section.

271 The $(\mathrm{Crl})$ of the nanopapers decreased when the WNFs were added. The XRD diffractograms 272 used to calculate the $\mathrm{Crl}$ are presented in Figure S3a. 100CNF has a Crl of $71 \%$; on the other 273 hand, 100WNF has $\mathrm{Crl}$ of 56\%. The hybrid nanopapers have a Crl value between of $100 \mathrm{CNF}$ and 274 100WNF, as expected (Figure S3b). The presence of amorphous lignin and hemicelluloses in 275 100WNF is the reason for the observed low Crl of WNF. Also, it has been reported that delignified 276 CNFs have higher crystallinity (Kaushik \& Singh, 2011). 

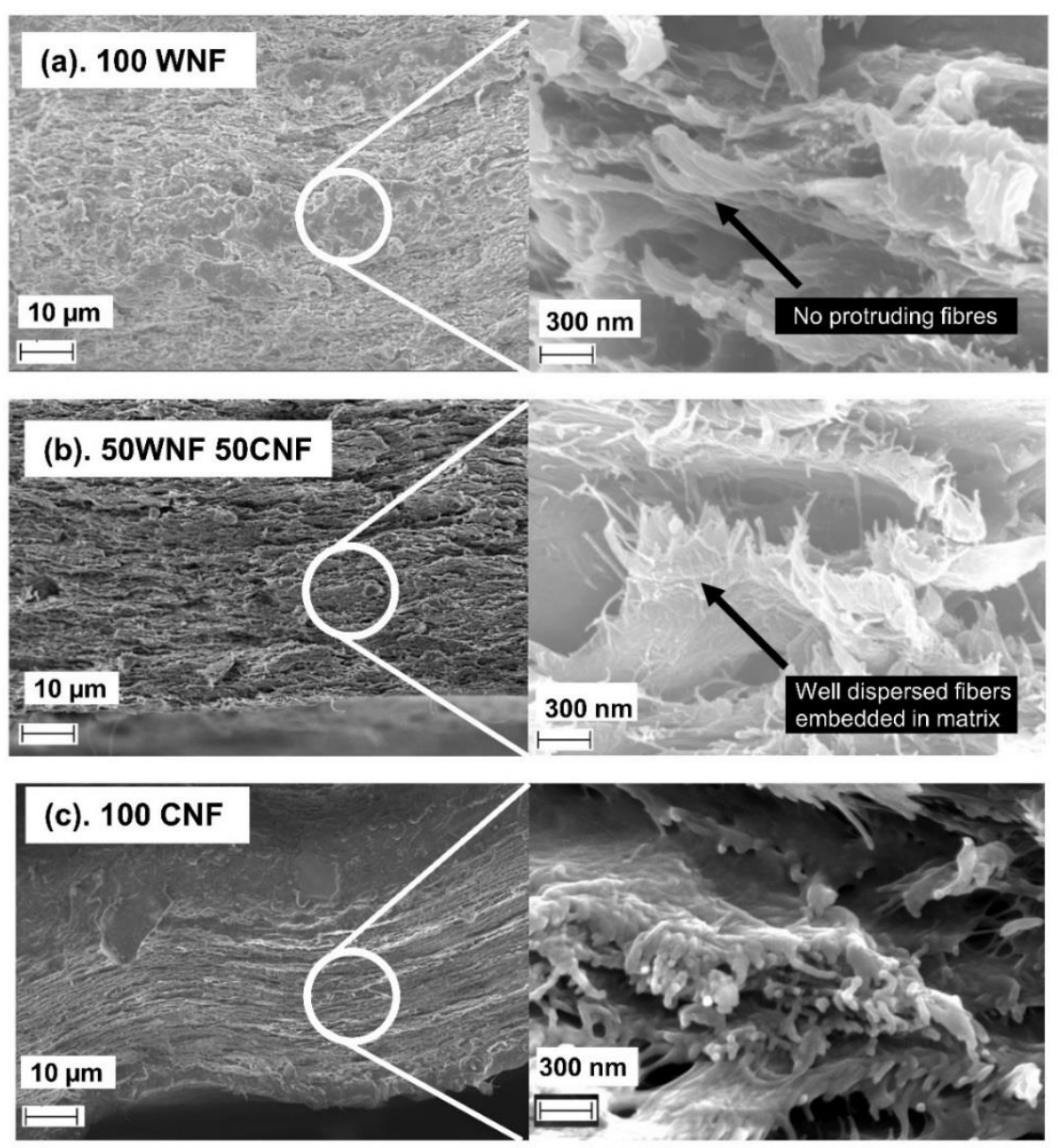

Figure 3. Cross-sectional fractured micrographs from the FESEM imaging of (a). 100WNF, (b). 50WNF50CNF, and (c). 100CNF. Images on right represent higher magnification of circled regions. In $100 \mathrm{WNF}$, layers are glued together with no fibres visible. In 100CNF and 50WNF50CNF, layers are distinct with fibres protruding out of the plane.

Adding the CNFs to the WNFs gave surprisingly good results. Testing found that $100 \mathrm{WNF}$ had the lowest mechanical properties (Figure 4); it was highly brittle with only $2.6 \%$ of elongation, a modulus of $4.3 \mathrm{GPa}$, and a tensile strength of $68 \mathrm{MPa}$. Hybrid nanopapers have higher modulus, 286 yield strength, tensile strength and elongation, compared to to 100WNF (Figure 4). The 287 quantitative results are presented in Table 2. Even at $30 \mathrm{wt} .-\%$ of added CNFs, the modulus of 288 hybrid nanopaper increased from 4.3 GPa to $5.4 \mathrm{GPa}$, an improvement of approximately $25 \%$. The 289 value of $5.4 \mathrm{GPa}$ is even higher than the theoretical value determined by the rule of mixtures (4.8 $290 \mathrm{GPa}$ ). Additionally, both the elongation and tensile strength improved by $80 \%$ and $50 \%$, 291 respectively, and an improvement of $33 \%$ in yield strength was also observed. 
292 The lower mechanical properties of $100 \mathrm{WNF}$ are due to the high concentration of lignin (27 wt.-\%). 293 It has been reported that lignin has a modulus of $3 \mathrm{GPa}$ and strength of $50 \mathrm{MPa}$ (Gibson, 2012), 294 which is significantly less than the properties of neat CNF networks (Figure 4). Another reason for 295 the reduced mechanical properties of 100WNF is the decrease in the extent of hydrogen bonding. 296 It has been reported that lignin interferes with hydrogen bonding (Horseman, Tajvidi, Diop, \& 297 Gardner, 2017; Rojo et al., 2015). WNFs are covered with lignin (Figure 2); hence, cellulose 298 entities are not free to form hydrogen bonds among each other. Hydrogen bonds are a strong bond 299 and the primary reason for the excellent dry mechanical properties of cellulosic nanopapers. 300 Additionally, hydrogen bonding also determines the yield strength (Benítez et al., 2013). Due to 301 lack of hydrogen bonding and tightly glueing of nanofibres by lignin, $100 \mathrm{WNF}$ yielded to $20 \mathrm{MPa}$ 302 before 100CNF. Finally, after yielding, 100WNF fractured abruptly, but 100CNF (and hybrid 303 nanopapers) exhibited a secondary inelastic elongation phase, where the deformation was 304 extended without fracturing due to interfibrillar sliding.

305 The results from hybrid nanopapers show a remarkable reinforcing ability of CNFs when added to 306 a WNF network. Another reason for such an improvement in mechanical properties is the formation 307 of a hydrogen-bonded network among CNFs. Apparently, a critical concentration is needed for 308 CNFs to percolate as no improvement was observed in hybrid nanopaper when the concentration 309 of CNF was 10 wt.-\%; however, at a concentration of 30 wt.- $\%$ and higher, the mechanical 310 properties showed improvement. Perhaps, at $10 \mathrm{wt} . \%$ CNFs were not able to make a network, but 311 at 30 wt.-\% the concentration was sufficient to make a stiff hydrogen-bonded network among 312 WNFs. The FESEM images also confirm this premise. The hybrid nanopaper has separated 313 individualised CNFs protruding out of the plane, interconnected to each other and forming a 314 network (Figure 3b). This hydrogen-bonded network is primarily responsible for the excellent 315 mechanical properties of nanopaper (Benítez et al., 2013) and interfibrillar sliding between CNFs is 316 responsible for the higher elongation (Benítez et al., 2013) Finally, an interesting observation can 317 be made from the stress-strain curves (Figure 4). The modulus and yield strength of hybrid 
nanopapers plateaued when the CNF concentration was above 30 wt.-\%. In contrast, the elongation and tensile strength increases with the increase in CNF concentration.

320 It can be concluded that entirely eliminating hydrogen bonding is not a wise decision and the 321 results can be seen in the stress-strain curves of nanopapers. Peresin et al. also concluded that it 322 is judicious to hydrophobise the surface of nanopaper, as modifying individual nanofibres interferes 323 with the hydrogen bonding (Peresin et al., 2017). This step is critical to imparting the mechanical properties of nanopaper. The hydroxyl groups of cellulose, which provide excellent mechanical properties by bonding to each other, are also responsible for their poor mechanical properties in the presence of water (Benítez et al., 2013). Therefore, a balance is highly desirable where hydrogen bonding can be controlled.

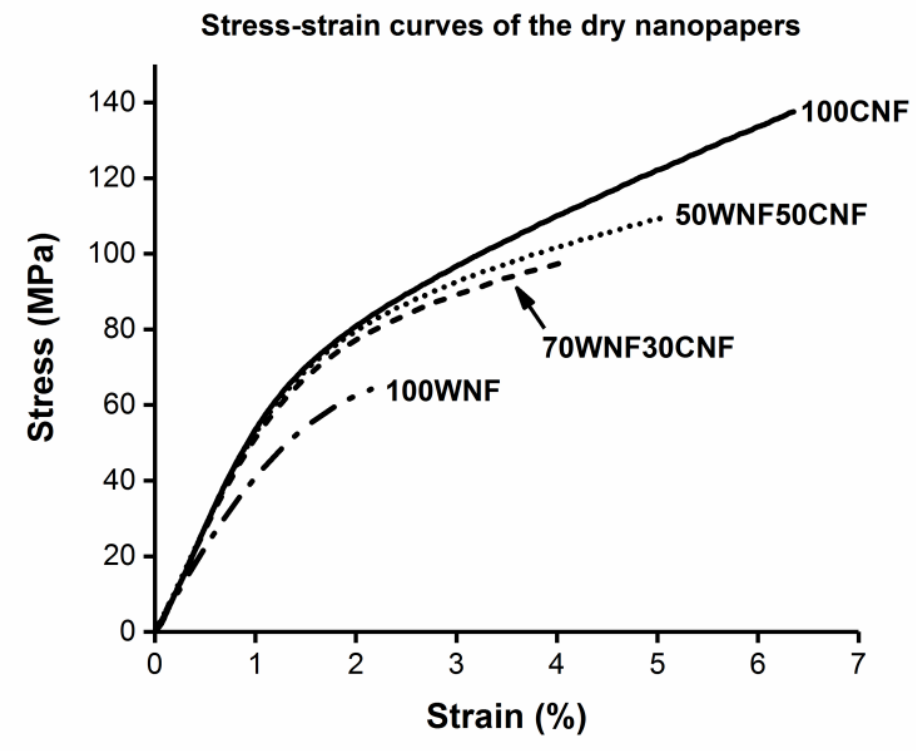

Figure 4. Stress-strain curves of dry nanopapers; $100 \mathrm{WNF}$ has the poorest properties, which improved considerably when CNFs were added. The elastic modulus, elongation, tensile and yield strength were also improved.

Table 2. Quantitative results from stress-strain analyses of references and hybrid nanopapers

\begin{tabular}{ccccc}
\hline Sample & $\begin{array}{c}\text { Elastic modulus } \\
(\mathrm{GPa})\end{array}$ & $\begin{array}{c}\text { Tensile Strength } \\
(\mathrm{MPa})\end{array}$ & $\begin{array}{c}\text { Elongation } \\
(\%)\end{array}$ & $\begin{array}{c}\text { Density } \\
\mathrm{g} / \mathrm{cm}^{3}\end{array}$ \\
\hline 100WNF & $4.3 \pm 0.1$ & $68 \pm 2$ & $2.6 \pm 0.1$ & 1.03 \\
\hline 90WNF10CNF & $4.3 \pm 0.1$ & $75 \pm 7$ & $2.1 \pm 0.3$ & 1.04 \\
\hline 70WNF30CNF & $5.4 \pm 0.2$ & $100 \pm 3$ & $4.75 \pm 0.3$ & 1.10 \\
\hline 50WNF50CNF & $5.5 \pm 0.2$ & $107 \pm 5$ & $5.3 \pm 06$ & 1.13 \\
\hline 30WNF70CNF & $5.8 \pm 0.1$ & $131 \pm 8$ & $7.4 \pm 1$ & 1.18 \\
\hline 100CNF & $6 \pm 0.1$ & $138 \pm 4$ & $6.9 \pm 0.6$ & 1.22 \\
\hline
\end{tabular}


334 The results of tensile testing are quite exciting, and better than expected. With just 30 wt.- $\%$ CNFs, 335 the modulus and yield strength of nanopaper is at par with $100 \mathrm{CNF}$. This result makes a compelling argument in support of the hybridisation of CNFs and WNFs, as using relatively fewer

337 CNFs results in a disproportionate contribution to the enhancement of properties. After considering

338 the draining time, 70WNF30CNF appeared to have good optimisation, comparable to the 339 mechanical properties of $100 \mathrm{CNF}$, at a draining time of only $35 \mathrm{~min}$, in contrast with 100CNF, 340 which took $120 \mathrm{~min}$. This ratio maintains a balance between the draining time and mechanical 341 properties of nanopapers, making it suitable for cost- effective production.

\subsection{Contact angle and water absorption}

343 Due to the presence of lignin, the hybrid nanopapers were more water repellent than 100CNF. The contact angle values, along with photographic images, are presented in Figure 5. Due to the high hydrophilicity of CNFs, $100 \mathrm{CNF}$ had a contact angle of $52^{\circ}$ with water. On the other hand, $100 \mathrm{WNF}$ had an angle of $107^{\circ}$, indicating it is highly hydrophobic. Between them, $70 \mathrm{WNF30CNF}$ had a contact angle of $95^{\circ}$, making it hydrophobic, as a surface demonstrating a contact angle of more than $90^{\circ}$ is considered hydrophobic. Clearly, lignin is effectively altering the surface, which can also be confirmed by FESEM images (Figure 2). The lignin has melted and fused, and, as a result, the hydrophilic CNFs are not in direct contact with the water droplet, causing the surface to become water repellent. It is worth mentioning that the contact angle of $30 \mathrm{WNF} 70 \mathrm{CNF}$ remains $25^{\circ}$ higher than $100 \mathrm{CNF}$, indicating that, even in a small quantity (8 wt.-\% in this case), the lignin, if uniformly distributed, can make a surface water repellent.

Making cellulosic materials hydrophobic is one of the biggest challenges for researchers. The most common technique is a surface modification using polymeric grafting, which is tedious and requires significant capital investment in reagents, solvents and chemical equipment. Paquet et al. grafted a high molecular weight polycaprolactone $\left(\mathrm{M}_{\mathrm{w}}=42,500\right)$ on CNF surface, using isocyanate-based grafting in methylene chloride and anhydrous toluene as a reaction medium to obtain a contact 
angle of $95^{\circ}$ (Paquet, Krouit, Bras, Thielemans, \& Belgacem, 2010). Other methods for

360 improvement of water repellency have also been reported. A contact angle of $70^{\circ}$ was achieved by 361 silylating the nanopaper surface (Peresin et al., 2017), whereas contact angles as high as $140^{\circ}$ 362 have been reported using the layer by layer deposition of carnauba wax (Forsman et al., 2017).

363 Our method gave a contact angle of $95^{\circ}$ with a simplistic eco-friendly approach, using cheaper raw 364 material and processing techniques known to the paper and pulp industry. This insight gives a rare, 365 lucrative advantage to the reported material.

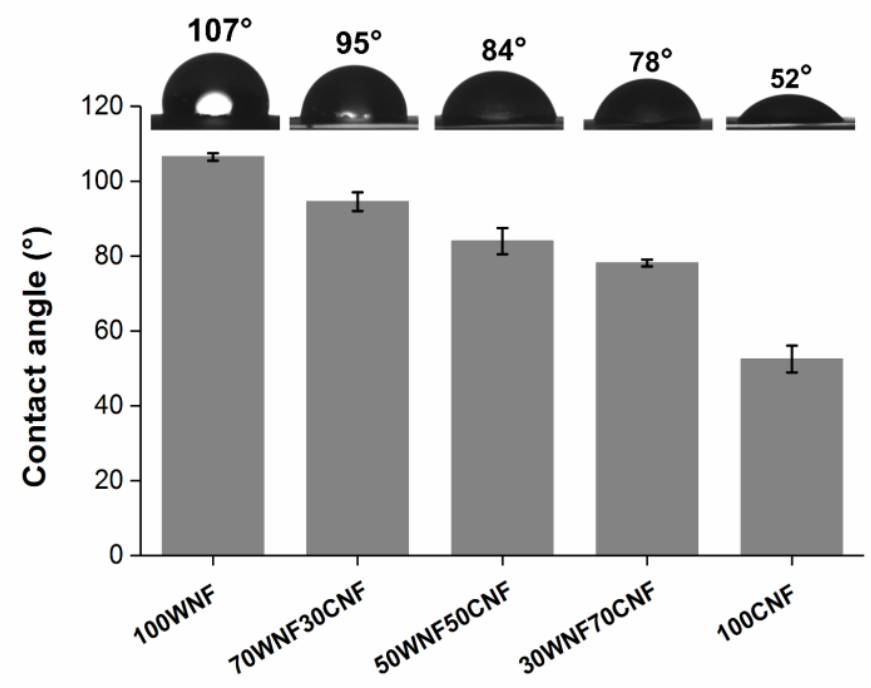

Figure 5. Water contact angle of reference and hybrid nanopapers; hybrid nanopapers are significantly more water repellent than $100 \mathrm{CNF}$, with $70 \mathrm{WNF30CNF}$ demonstrating hydrophobicity.

369 Hybrid nanopapers not only had a higher contact angle, but they were also relatively impervious to water (Figure S4a); 100WNF absorbed the least amount of water. After soaking for 24 hours,

371100 CNF absorbed $110 \%$ of water; on the other hand, 100 WNF absorbed $69 \%$ of water. The

372 magnitude of water absorbed by hybrid nanopapers was intermediate to that of $100 \mathrm{CNF}$ and $373100 \mathrm{WNF}$. It can be concluded that the presence of lignin makes nanopaper resistant to water 374 absorption. Interestingly, there appears to be a linear relationship between the amount of lignin and water absorption (Figure S4b). The higher the amount of lignin in nanopaper, the less water it

376 absorbed when dipped in water. It has been reported that lignin fills the voids and crosslinks with 377 the cellulose, which causes a decrease in water absorption (Nair \& Yan, 2015). 


\subsection{Wet mechanical properties}

379 When wet, $100 \mathrm{CNF}$ weakened the most and retained only $15 \%$ of its dry modulus (1 GPa when wet, from $6 \mathrm{GPa}$ when dry; see Figure 6b). Additionally, it exhibited no yield point (Figure 6a), which indicates that it yielded right away to the inelastic region and permanently deformed as soon as a load was applied, demonstrating that the presence of water causes significant structural changes in nanopaper. Firstly, water seeps into the interfibrillar regions, diminishing the hydrogen bonding (Benítez et al., 2013), which is the primary factor responsible for stiffness. Once the hydrogen bonding is gone, the nanopaper permanently deforms under a considerably lower load than it would when dry (as seen in Figure 6). Secondly, water molecules plasticise the hemicelluloses, which are present in the non-crystalline part of CNFs (Cunha, Zhou, Larsson, \& Berglund, 2014), and, hence, the modulus is decreased.

Lignin-rich nanopapers, on the other hand, were able to resist the water. As measured, 100WNF retained $75 \%$ of its modulus (3.2 GPa when wet from $4.3 \mathrm{GPa}$ when dry), which was $220 \%$ more than wet 100CNF. Additionally, 100WNF showed elastic behaviour in the elastic region and yield, which are marks of a stiff material. Hybrid nanopaper also benefitted from the presence of lignin and showed improved mechanical properties when wet (Figure $6 \mathrm{a}$ and b). While wet, 70WNF30CNF retained $40 \%$ of its modulus (100CNF retained $15 \%$ ) and showed elastic behaviour in the beginning. The presence of lignin restricts interfibrillar sliding, as lignin has melted and fused with fibres (Figure 2), which maintains the integrity of nanopaper even when damp. These results are considerably better than other reported studies (Lucenius et al., 2014; Sehaqui et al., 2014).

398 Another significant finding is that the mechanical properties of $100 \mathrm{WNF}$ are still in the range 399 representing engineering polymers (modulus 3.5 GPa and strength $38 \mathrm{MPa}$ ). Water is an abundant 400 material that frequently interacts with everyday materials, and a product such as nanopaper is 401 expected to display some considerable amount of water resistance. Doing so is the only way to 402 safeguard the commercialisation of nanopaper. 

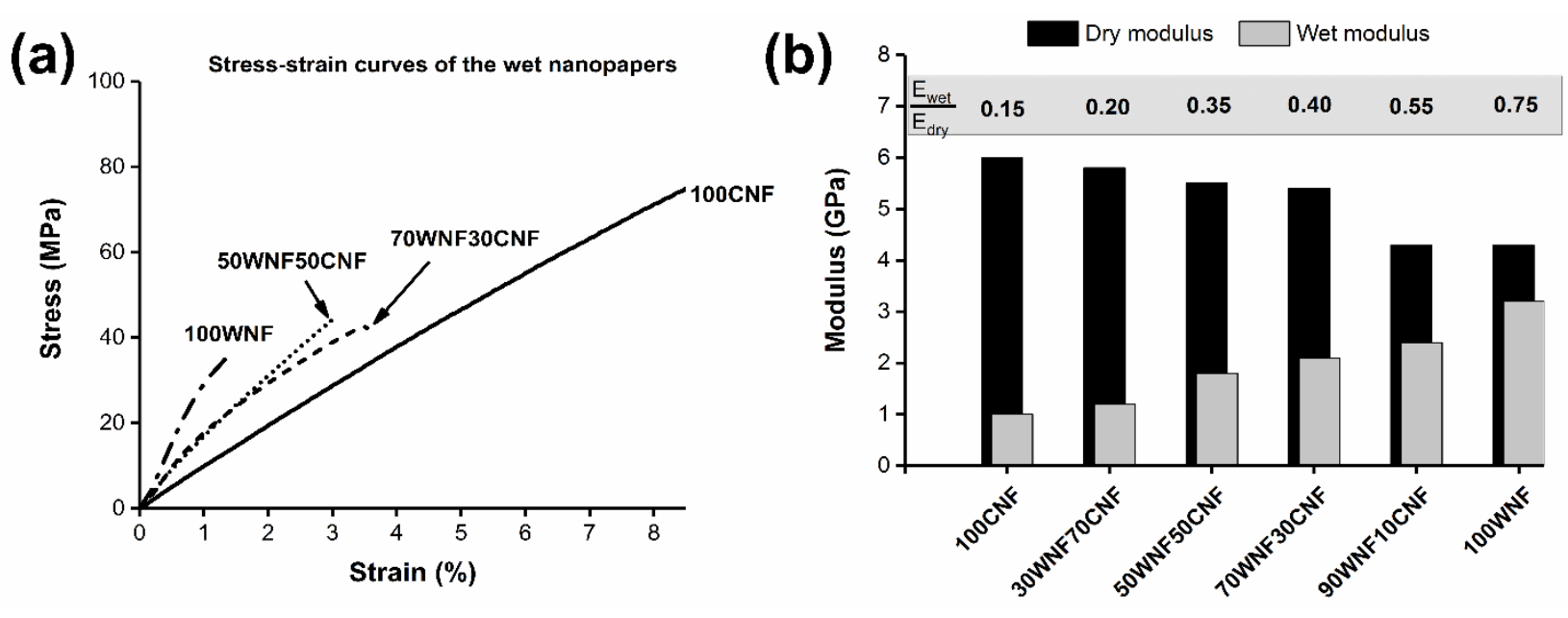

Figure 6. (a) Stress-strain curves after wetting the reference and hybrid nanopapers; 100CNF yielded right away and lost its stiffness, while 100WNF retained its stiffness. Hybrid nanopapers have better wet properties than 100CNFs. (b) Comparison of dry and wet modulus of reference and hybrid nanopapers; $100 \mathrm{CNF}$ lost $85 \%$ of its modulus, while $100 \mathrm{WNF}$ lost only $25 \%$. Hybrid nanopapers displayed intermediate modulus to $100 \mathrm{CNF}$ and $100 \mathrm{WNF}$.

\subsection{Effect of Humidity}

The moisture absorbed from humid conditions is equally damaging to the mechanical properties of $100 \mathrm{CNF}$ as soaking it in water; $100 \mathrm{CNF}$ lost $87 \%$ of its stiffness (storage modulus) in the presence of $95 \% \mathrm{RH}$, which is the humidity in the atmosphere on a rainy day. In contrast, the hybrid nanopapers had a higher tolerance towards humidity than both 100CNF and 100WNF (Figure 7). In the first stage of the experiment (when the humidity was steadily increased from $0 \%$ to $95 \%$ $\mathrm{RH})$, all the nanopapers showed a similar response, which included an initial stiff phase where the modulus showed no sudden fluctuation. After the humidity reached around $60-70 \% \mathrm{RH}$, the modulus started dropping steadily. When the humidity reached 95\%, 100CNF still had a higher modulus than 100WNF and hybrid nanopapers. It was only in the second stage, when the humidity was kept constant at $95 \% \mathrm{RH}$, that $100 \mathrm{CNF}$ lost its storage modulus in two sudden linear drops;no such behaviour was observed with 100WNF and hybrid nanopapers. In the end, the storage modulus of $100 \mathrm{CNF}$ reached a plateau value corresponding to a loss of $87 \%$ in its stiffness, which was, unexpectedly, the same drop as observed in the elastic modulus of wet tensile testing results (Figure 6b). On the other hand, 100WNF and hybrid nanopapers did not show any sudden drops and quickly reached a plateau value, which did not decrease any further, no matter how long the sample was exposed to the humidity. This result indicates that moisture weakens the materials a 
bit, but the nanopapers were able to maintain their integrity. Finally, nanopapers containing WNFs lost around $60-65 \%$ of their properties (100CNF lost $87 \%$ ). The advantage of hybridisation can be explained briefly by the results from 50WNF50CNF. After equilibrium, it had 2.3 times the modulus of $100 \mathrm{CNF}$ and 1.5 times the modulus of $100 \mathrm{WNF}$. This difference shows the promising value of such materials, which have a high quantity of moisture-susceptible material, yet the hybrid nanopapers showed as much resistance as 100WNF. Apart from the magnitude of the storage modulus, it was found that incorporating lignin fibres delayed the onset of losing storage modulus.

433 The onset point for $100 \mathrm{WNF}$ was $77 \% \mathrm{RH}$, while for $100 \mathrm{CNF}$ it was $70 \% \mathrm{RH}$, indicating the stable nature of 100WNF, which was transferred to hybrid nanopapers. The onset point for 70WNF30CNF was $75 \% \mathrm{RH}$ and for $50 \mathrm{WNF50CNF}$ was $73 \%$.

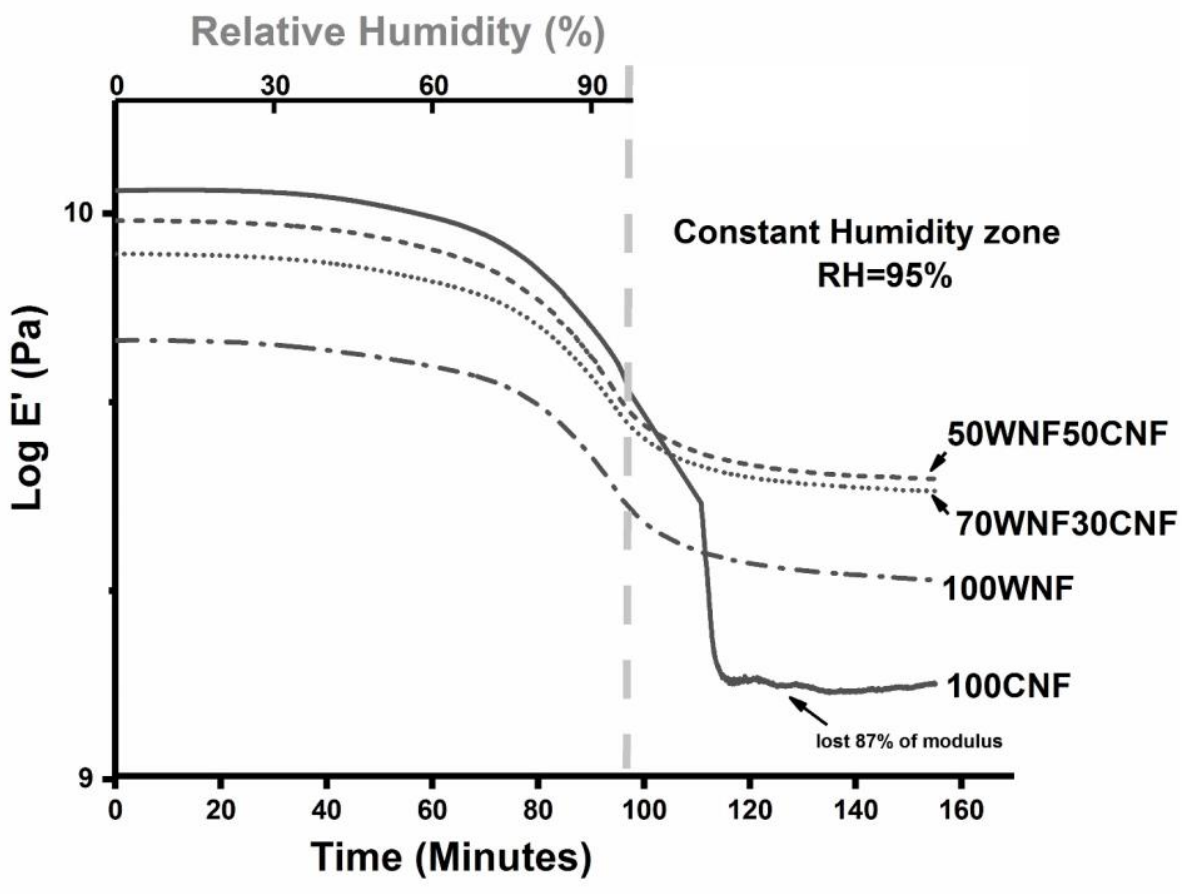

Figure 7 Variations in the storage modulus of nanopapers with the variations in relative humidity. Nanopapers with WNF resisted the humid conditions, while 100CNF lost most of its stiffness.

440 The deterioration of storage modulus in the presence of moisture can be explained by the

441 disruption of hydrogen bonding (Sethi et al., 2018) and plasticisation of amorphous regions 442 (Benítez et al., 2013). It has been reported that nanopapers can absorb up to $30 \%$ water at 95\%RH (Benítez et al., 2013). Such a large amount of water leads to dimensional changes and 
swelling, and, as hydrogen bonds are weakened, the nanopapers lose their modulus. The presence of lignin makes the nanopaper impervious to the environment; thus, they resist the impact of humid environments. Moisture resistance is one of the mandatory properties for the success of commercial materials. If nanocellulosic materials are to be commercialised in the near future, this issue needs to be adequately addressed. Even in the presence of high humidity, the CNF nanopapers lose significant stiffness. Hybridisation with wood fibres can be one of the potential ways to improve it.

\subsection{Thermal stability}

The chemical composition of 100WNF caused it to have the poorest thermal stability out of all the nanopapers (until $350^{\circ} \mathrm{C}$ ). WNF consists of many materials, such as lignin hemicellulose, which are extractives that are known to intensify degradation (Poletto, Zattera, Forte, \& Santana, 2012). On the other hand, 100CNF has better thermal stability than 100WNF because it has higher crystallinity(Kim, Eom, \& Wada, 2010). Interestingly, the hybrid nanopaper (50WNF50CNF) had a thermal stability superior to both 100CNF and 100WNF (Figure 8). By the time the temperature reached $340^{\circ} \mathrm{C}, 50 \mathrm{WNF} 50 \mathrm{CNF}$ had degraded $29 \%$, while $100 \mathrm{WNF}$ and $100 \mathrm{CNF}$ degraded $31 \%$ and $37 \%$ of their weight, respectively. Additionally, it is worth mentioning that the thermal stability of $100 \mathrm{WNF}$ was inferior only until $350^{\circ} \mathrm{C}$. At temperatures higher than that, $100 \mathrm{WNF}$ became more stable than 50WNF50CNF (100CNF remained thermally inferior) (Figure S5). The reason is the slower degradation rate of lignin and stable by-products, which were also indicated by the amount of char left in the end. According to measurements, $100 \mathrm{WNF}$ left $24 \%$ char, $50 \mathrm{WNF} 50 \mathrm{CNF}$, left $22 \%$ char and 100 CNF left $19 \%$ char.

The improved thermal stability of hybrid nanopapers should be credited to the interpenetrating morphology, which is known to impart thermal stability to materials (Sethi, Illikainen, Sain, \& Oksman, 2017). Combining materials creates more interfaces, which delays the effective heat transfer and, in turn, delaying the degradation. It has been reported that increasing the interactions improves the thermal stability (Fu et al., 2008). Hybridisation conclusively benefits the thermal stability of nanopapers, which is necessary for high-temperature processing and long-term use in 
471 flexible circuits, which can overheat due to electricity. Increased thermal stability is one advantage 472 of the reported procedure, as it uses non-functionalised fibres, due to which it is able to better 473 maintain (and even enhance) thermal properties. It has been reported that chemical 474 functionalisation leads to a decrease in thermal stability (Fukuzumi, Saito, Iwata, Kumamoto, \& 475 Isogai, 2009)(Eyholzer et al., 2010), and it uses expensive reagents and equipment, making it 476 fiscally burdensome.

(a)

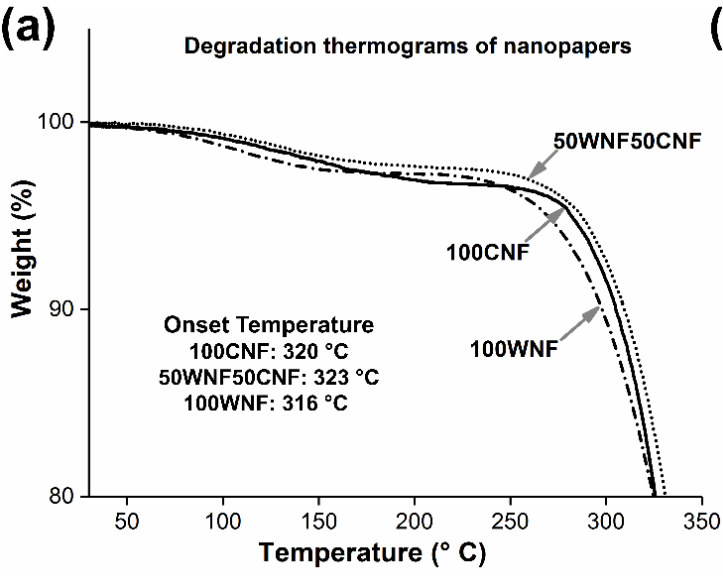

(b)

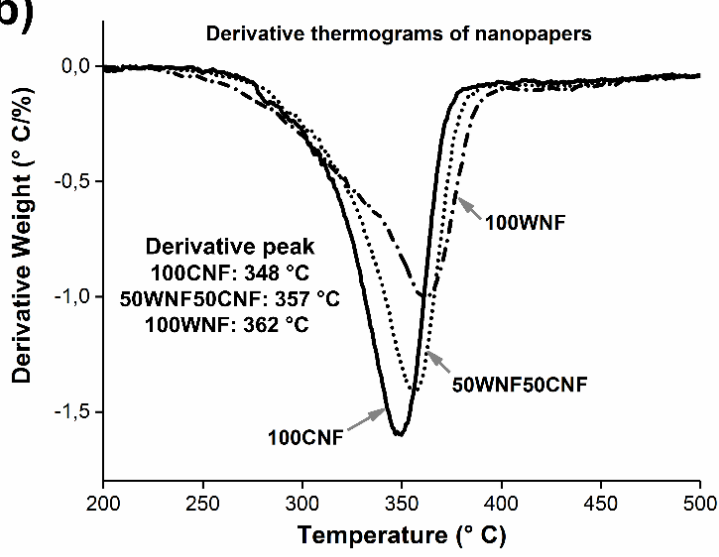

Figure 8 Degradation and derivative thermogram of 100WNF, 50WNF50CNF and 100CNF. 50WNF50CNF is most thermally stable.

\section{Conclusions}

In this study, we argue that the presence of lignin in nanopapers can help improve their properties and suggest a simple method to prepare lignin-rich hybrid nanopapers from WNF and CNF. It was found that in tandem, WNF and CNF behave synergistically to compensate for the drawbacks of each other. The synergistic effect was strong and many properties were better than what was expected by rule of mixture. The 70WNF30CNF hybrid nanopapers drained in 35 minutes, which was 85 minutes faster than 100CNF. Additionally, the hybrid nanopapers displayed significant improvement over the mechanical properties of 100WNF. Furthermore, due to the presence of lignin, the hybrid nanopaper showed improved mechanical properties under the influence water and humidity (in comparison to 100CNF). The reason for this improvement is that lignin melts and fuse with the nanocellulosic entities to bind them together. We present a method to obtain a highend, lignin-rich product without arduous procedures. It is a laborious task to separate lignin for it to 
be used in commercial products. (Yang, Zhong, Yuan, Peng, \& Sun, 2013). Further work on using 493 lignin-rich hybrid nanopaper for applications such as gas barrier membranes and other applications 494 is in the planning stage.

\section{Acknowledgement}

\section{References}

Benítez, A. J., Torres-Rendon, J., Poutanen, M., \& Walther, A. (2013). Humidity and multiscale structure govern mechanical properties and deformation modes in films of native cellulose nanofibrils. Biomacromolecules, 14(12), 4497-4506. http://doi.org/10.1021/bm401451m

Chun, S. J., Lee, S. Y., Doh, G. H., Lee, S., \& Kim, J. H. (2011). Preparation of ultrastrength nanopapers using cellulose nanofibrils. Journal of Industrial and Engineering Chemistry, 17(3), 521-526. http://doi.org/10.1016/j.jiec.2010.10.022

Cunha, A. G., Zhou, Q., Larsson, P. T., \& Berglund, L. A. (2014). Topochemical acetylation of cellulose nanopaper structures for biocomposites: mechanisms for reduced water vapour sorption. Cellulose, 21(4), 2773-2787. http://doi.org/10.1007/s10570-014-0334-z

Deng, S., Huang, R., Zhou, M., Chen, F., \& Fu, Q. (2016). Hydrophobic cellulose films with excellent strength and toughness via ball milling activated acylation of microfibrillated cellulose. Carbohydrate Polymers, 154, 129-138. http://doi.org/10.1016/j.carbpol.2016.07.101

Eyholzer, C., Bordeanu, N., Lopez-Suevos, F., Rentsch, D., Zimmermann, T., \& Oksman, K. (2010). Preparation and characterization of water-redispersible nanofibrillated cellulose in powder form. Cellulose, 17(1), 19-30. http://doi.org/10.1007/s10570-009-9372-3 
Forsman, N., Lozhechnikova, A., Khakalo, A., Johansson, L. S., Vartiainen, J., \& Österberg, M. (2017). Layer-by-layer assembled hydrophobic coatings for cellulose nanofibril films and textiles, made of polylysine and natural wax particles. Carbohydrate Polymers, 173, 392-402. http://doi.org/10.1016/j.carbpol.2017.06.007

French, A. D. (2014). Idealized powder diffraction patterns for cellulose polymorphs. Cellulose, 21(2), 885896. http://doi.org/10.1007/s10570-013-0030-4

Fu, J.-F., Shi, L.-Y., Yuan, S., Zhong, Q.-D., Zhang, D.-S., Chen, Y., \& Wu, J. (2008). Morphology, toughness mechanism, and thermal properties of hyperbranched epoxy modified diglycidyl ether of bisphenol A (DGEBA) interpenetrating polymer networks. Polymers for Advanced Technologies, 19(11), 1597-1607. http://doi.org/10.1002/pat.1175

Fukuzumi, H., Saito, T., Iwata, T., Kumamoto, Y., \& Isogai, A. (2009). Transparent and High Gas Barrier Films of Cellulose Nanofibers Prepared by TEMPO-Mediated Oxidation. Biomacromolecules, 10(1), 162-165. http://doi.org/10.1021/bm801065u

Gibson, L. J. (2012). The hierarchical structure and mechanics of plant materials. J. The Royal Soc. Interface, 9(76), pp 2749-2766. http://doi.org/10.1098/rsif.2012.0341

Henriksson, G. (2017). What are the biological functions of lignin and its complexation with carbohydrates? - OPEN ACCESS. Nordic Pulp and Paper Research Journal, 32(04), 527-541. http://doi.org/10.3183/NPPRJ-2017-32-04-p527-541

Henriksson, M., Berglund, L. A., Isaksson, P., Lindström, T., \& Nishino, T. (2008). Cellulose nanopaper structures of high toughness. Biomacromolecules, 9(6), 1579-1585. http://doi.org/10.1021/bm800038n

Herrera, M., Thitiwutthisakul, K., Yang, X., Rujitanaroj, P., Rojas, R., \& Berglund, L. (2018). Preparation and evaluation of high-lignin content cellulose nanofibrils from eucalyptus pulp. Cellulose. http://doi.org/10.1007/s10570-018-1764-9

Horseman, T., Tajvidi, M., Diop, C. I. K., \& Gardner, D. J. (2017). Preparation and property assessment of neat lignocellulose nanofibrils (LCNF) and their composite films. Cellulose, 24(6), 2455-2468. 
http://doi.org/10.1007/s10570-017-1266-1

Huang, W. (Ed.). (2017). Nanopapers: From Nanochemistry and Nanomanufacturing to Advanced Applications (1st Editio). Elsevier. Retrieved from https://www.elsevier.com/books/nanopapers/huang/978-0-323-48019-2

Iwamoto, S., Nakagaito, a. N., Yano, H., \& Nogi, M. (2005). Optically transparent composites reinforced with plant fiber-based nanofibers. Applied Physics A: Materials Science and Processing, 81(6), 11091112. http://doi.org/10.1007/s00339-005-3316-z

Kargarzadeh, H., Ahmad, I., Thomas, S., \& Dufresne, A. (2017). Handbook of Nanocellulose and Cellulose Nanocomposites, 2 Volume Set. John Wiley \& Sons.

Kaushik, A., \& Singh, M. (2011). Isolation and characterization of cellulose nanofibrils from wheat straw using steam explosion coupled with high shear homogenization. Carbohydrate Research, 346(1), 7685. http://doi.org/10.1016/j.carres.2010.10.020

Kim, U. J., Eom, S. H., \& Wada, M. (2010). Thermal decomposition of native cellulose: Influence on crystallite size. Polymer Degradation and Stability, 95(5), 778-781. http://doi.org/10.1016/j.polymdegradstab.2010.02.009

Koga, H., Nogi, M., Komoda, N., Nge, T. T., Sugahara, T., \& Suganuma, K. (2014). Uniformly connected conductive networks on cellulose nanofiber paper for transparent paper electronics. NPG Asia Materials, 6(3), e93. http://doi.org/10.1038/am.2014.9

Kubo, S., \& Kadla, J. F. (2005). Hydrogen bonding in lignin: A fourier transform infrared model compound study. Biomacromolecules, 6(5), 2815-2821. http://doi.org/10.1021/bm050288q

Liu, A., Walther, A., Ikkala, O., Belova, L., \& Berglund, L. A. (2011). Clay nanopaper with tough cellulose nanofiber matrix for fire retardancy and gas barrier functions. Biomacromolecules, 12(3), 633-641. http://doi.org/10.1021/bm101296z

Lucenius, J., Parikka, K., \& Österberg, M. (2014). Nanocomposite films based on cellulose nanofibrils and water-soluble polysaccharides. Reactive and Functional Polymers, 85, 167-174. 
http://doi.org/10.1016/j.reactfunctpolym.2014.08.001

Mazeau, K., \& Rivet, A. (2008). Wetting the (110) and (100) surfaces of Ibeta cellulose studied by molecular dynamics. Biomacromolecules, 9(4), 1352-4. http://doi.org/10.1021/bm7013872

Nair, S. S., \& Yan, N. (2015). Effect of high residual lignin on the thermal stability of nanofibrils and its enhanced mechanical performance in aqueous environments. Cellulose, 22(5), 3137-3150. http://doi.org/10.1007/s10570-015-0737-5

Österberg, M., Vartiainen, J., Lucenius, J., Hippi, U., Seppälä, J., Serimaa, R., \& Laine, J. (2013). A fast method to produce strong NFC films as a platform for barrier and functional materials. ACS Applied Materials and Interfaces, 5(11), 4640-4647. http://doi.org/10.1021/am401046x

Paquet, O., Krouit, M., Bras, J., Thielemans, W., \& Belgacem, M. N. (2010). Surface modification of cellulose by PCL grafts. Acta Materialia, 58(3), 792-801. http://doi.org/10.1016/j.actamat.2009.09.057

Peresin, M. S., Kammiovirta, K., Heikkinen, H., Johansson, L. S., Vartiainen, J., Setälä, H., ... Tammelin, T. (2017). Understanding the mechanisms of oxygen diffusion through surface functionalized nanocellulose films. Carbohydrate Polymers, 174, 309-317. http://doi.org/10.1016/j.carbpol.2017.06.066

Poletto, M., Zattera, A. J., Forte, M. M. C., \& Santana, R. M. C. (2012). Thermal decomposition of wood: Influence of wood components and cellulose crystallite size. Bioresource Technology, 109, 148-153. http://doi.org/10.1016/j.biortech.2011.11.122

Rojo, E., Peresin, M. S., Sampson, W. W., Hoeger, I. C., Vartiainen, J., Laine, J., \& Rojas, O. J. (2015). Comprehensive elucidation of the effect of residual lignin on the physical, barrier, mechanical and surface properties of nanocellulose films. Green Chem., 17(3), 1853-1866. http://doi.org/10.1039/C4GC02398F

Salajkova, M., Valentini, L., Zhou, Q., \& Berglund, L. A. (2013). Tough nanopaper structures based on cellulose nanofibers and carbon nanotubes. Composites Science and Technology, 87, 103-110. http://doi.org/10.1016/j.compscitech.2013.06.014 
Sehaqui, H., Ezekiel Mushi, N., Morimune, S., Salajkova, M., Nishino, T., \& Berglund, L. A. (2012). Cellulose nanofiber orientation in nanopaper and nanocomposites by cold drawing. ACS Applied Materials and Interfaces, 4(2), 1043-1049. http://doi.org/10.1021/am2016766

Sehaqui, H., Kochumalayil, J., Liu, A., Zimmermann, T., \& Berglund, L. A. (2013). Multifunctional nanoclay hybrids of high toughness, thermal, and barrier performances. ACS Applied Materials and Interfaces, 5(15), 7613-7620. http://doi.org/10.1021/am401928d

Sehaqui, H., Liu, A., Zhou, Q., \& Berglund, L. A. (2010). Fast preparation procedure for large, flat cellulose and cellulose/inorganic nanopaper structures. Biomacromolecules, 11(9), 2195-2198. http://doi.org/10.1021/bm100490s

Sehaqui, H., Zimmermann, T., \& Tingaut, P. (2014). Hydrophobic cellulose nanopaper through a mild esterification procedure. Cellulose, 21(1), 367-382. http://doi.org/10.1007/s10570-013-0110-5

Sethi, J., Farooq, M., Sain, S., Sain, M., Sirviö, J. A., Illikainen, M., \& Oksman, K. (2018). Water resistant nanopapers prepared by lactic acid modified cellulose nanofibers. Cellulose, 25(1), 259-268. http://doi.org/10.1007/s10570-017-1540-2

Sethi, J., Illikainen, M., Sain, M., \& Oksman, K. (2017). Polylactic acid/polyurethane blend reinforced with cellulose nanocrystals with semi-interpenetrating polymer network (S-IPN) structure. European Polymer Journal, 86, 188-199. http://doi.org/http://dx.doi.org/10.1016/j.eurpolymj.2016.11.031

Tenhunen, T., Hakalahti, M., Kouko, J., Salminen, A., Härkäsalmi, T., Pere, J., ... Hänninen, T. (2016). Method for Forming Pulp Fibre Yarns Developed by a by a Design-driven Process. BioResources, 11(1), 2492-2503.

Visanko, M., Sirviö, J. A., Piltonen, P., Sliz, R., Liimatainen, H., \& Illikainen, M. (2017). Mechanical fabrication of high-strength and redispersible wood nanofibers from unbleached groundwood pulp. Cellulose, 44, 4173-4187. http://doi.org/10.1007/s10570-017-1406-7

Wang, X., Hu, J., \& Zeng, J. (2012). Steam explosion pulping of oil palm empty fruit bunch fiber. BioResources, 7(1), 1008-1015. 
616 Yagyu, H., Ifuku, S., \& Nogi, M. (2017). Acetylation of optically transparent cellulose nanopaper for high 617 thermal and moisture resistance in a flexible device substrate. Flexible and Printed Electronics, 2(1). $618 \quad$ http://doi.org/10.1088/2058-8585/aa60f4

619 Yang, D., Zhong, L. X., Yuan, T. Q., Peng, X. W., \& Sun, R. C. (2013). Studies on the structural 620 characterization of lignin, hemicelluloses and cellulose fractionated by ionic liquid followed by alkaline 621 extraction from bamboo. Industrial Crops and Products, 43(1), 141-149.

622 http://doi.org/10.1016/j.indcrop.2012.07.024

623 